\title{
Bilateral Renal Papillary Hypertrophy: A Rare Cause of Benign Es- sential Hematuria Endoscopic Diagnosis and Management
}

\author{
${ }^{1}$ Eastern Virginia Medical School, Norfolk, VA, USA \\ ${ }^{2}$ Children's Hospital of The King's Daughters, USA
}

Alexander Birk ${ }^{1}$, Achanking Afiadata ${ }^{1 *}$ and Jyoti Upadhyay ${ }^{1,2}$

*Corresponding author: Achanking Afiadata, MD, Children's Hospital of The King's Daughters, USA, E-mail: afiadaa@EVMS.EDU

\begin{abstract}
Introduction: Benign essential hematuria is an idiopathic gross hematuria of renal origin without evidence of radiographic, histologic, or functional abnormalities of the kidney. Although the natural history for benign essential hematuria often is spontaneous resolution of symptom, some severe cases may require treatment for control of persistent hematuria.

The etiology of benign essential hematuria is not well elucidated. Unilateral renal papillary hypertrophy has been reported. Traditionally, management has included invasive treatment including nephrectomy or partial nephrectomy due to lack of correct diagnosis in the presence of hematuria.

We present two cases of essential hematuria associated with bilateral diffuse hypertrophy of the renal papillae.

To our knowledge, bilateral involvement has not been reported previously. In both of our adolescent female patients, conservative management with endoscopic diagnosis and treatment was successful.

Conclusion: Papillary hypertrophy is a rare anatomic abnormality of the kidney which can result in benign hematuria. Although the etiology of this entity is not clear, knowledge of the presentation is important to preserve the renal function and avoid unnecessary invasive treatment. Conservative approach with minimally invasive diagnosis and treatment is indicated as first line treatment.
\end{abstract}

\section{Introduction}

Benign essential hematuria is characterized by gross hematuria of renal origin without evidence of radiographic, histologic or functional abnormalities of the kidneys [1]. The hematuria can be acute, intermittent or chronic, and from a unilateral or bilateral source. It is also known as chronic unilateral hematuria, lateralizing essential hematuria, or benign lateralizing hematuria $[2,3]$.
It is a diagnosis of exclusion, and involves work-up with a complete history, physical examination, appropriate laboratory evaluation (complete blood count, coagulation studies, urinalysis with culture, and urine cytology), imaging studies (renal and bladder ultrasound, CT urogram, magnetic resonance urogram, retrograde pyelogram, and possible renal angiography), and cystoscopy [3]. Some of the more common reported causes include venous rupture, hemangioma on renal papilla, and calculus [3]. Renal papillary hypertrophy has been reported as one cause of benign hematuria. In the majority of cases, the disease has a benign course and the hematuria resolves in most patients without intervention. However, in some patients with intractable hematuria and symptomatic anemia, partial or complete nephrectomy has been performed previously [2].

With advancement in technology and availability of smaller instruments, endoscopy with laser ablation or fulguration of lesions, if present, is now the preferred treatment when the preoperative diagnosis is apparent. This paper describes two cases of adolescent patients with papillary hypertrophy as the cause of essential hematuria.

\section{Case 1 - EC}

EC is a 15-year-old female patient who presented with 3 months of persistent painless hematuria presenting as daily episodes of bright-red to cola-colored urine. She has used hormonal birth control for over 6 months to help control her heavy menstrual periods. Patient was normotensive with an unremarkable physical exam. Initial work-up with complete blood count, serum chemistries, coagulation studies and complement studies were with-

Citation: Birk A, Afiadata A, Upadhyay J (2017) Bilateral Renal Papillary Hypertrophy: A Rare Cause of Benign Essential Hematuria Endoscopic Diagnosis and Management. Int Arch Urol Complic 3:031. doi. org/10.23937/2469-5742/1510031

Received: July 24, 2017: Accepted: September 28, 2017: Published: September 30, 2017

Copyright: (C) 2017 Birk A, et al. This is an open-access article distributed under the terms of the Creative Commons Attribution License, which permits unrestricted use, distribution, and reproduction in any medium, provided the original author and source are credited.

INTERNATIONALISE

INTERNATIONAL LIBRARY 


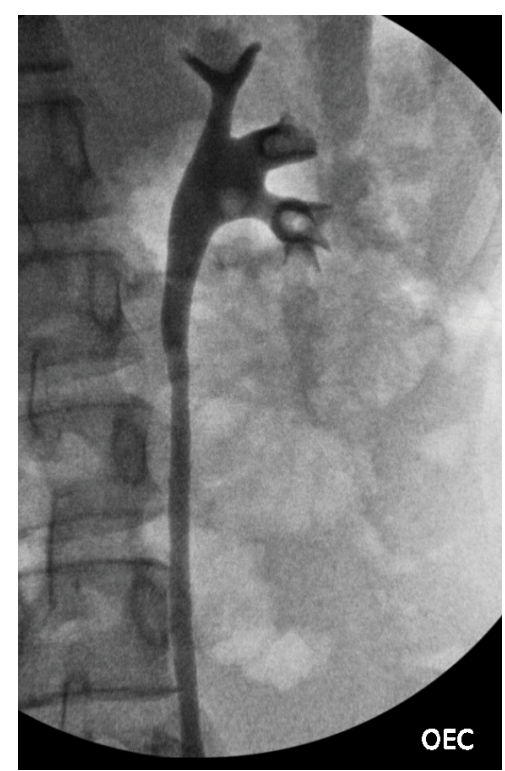

A

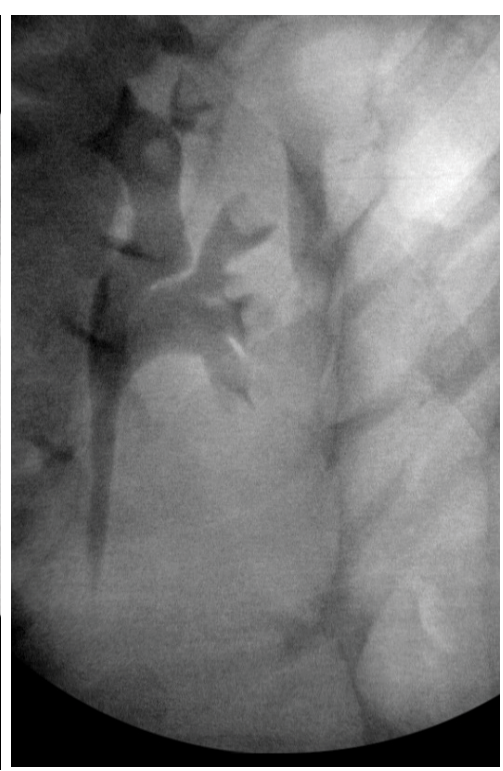

B

Figure 1a: Left retrograde pyelogram demonstrating filling defects in renal pelvis, lower pole and mid pole calyces. Case 1-EC.

Figure 1b: Left retrograde pyelogram demonstrating filling defect in left upper pole and mid pole calyces. Case 2-AS.
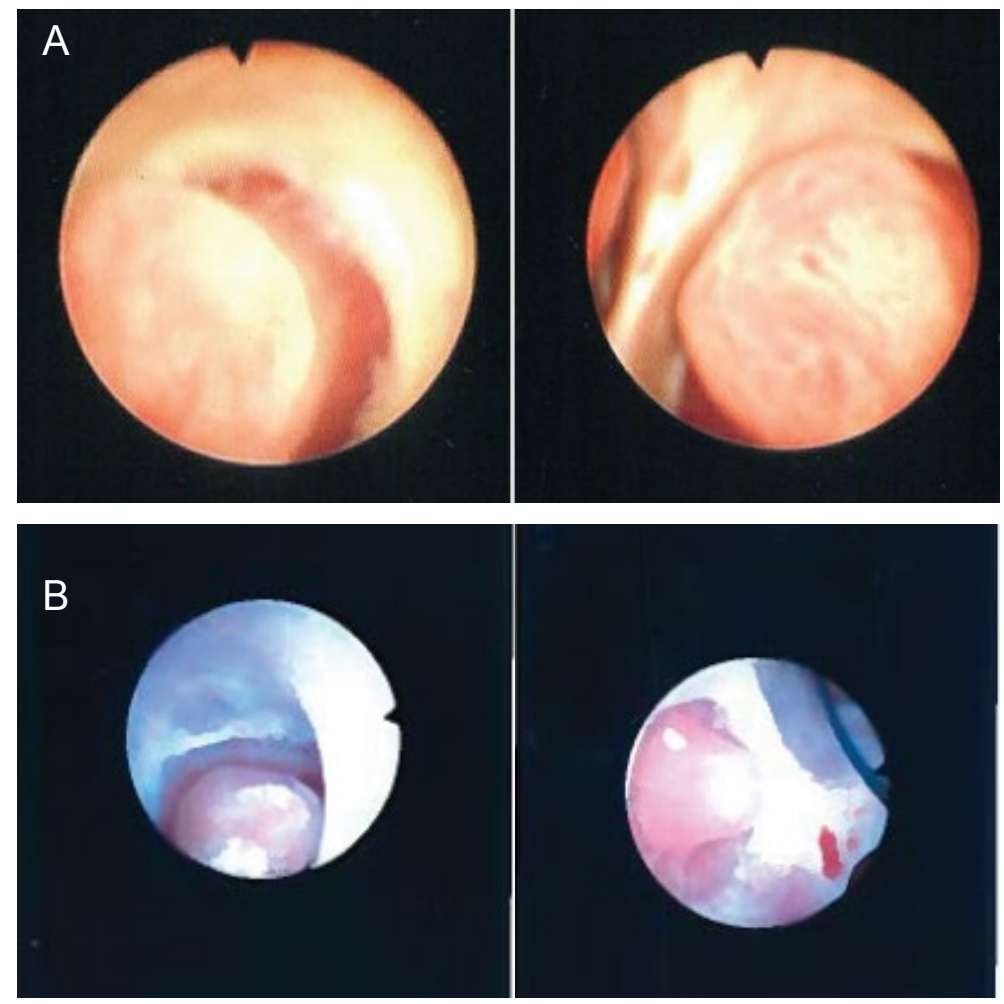

Figure 2a: Hypertrophied papilla of the left mid pole and lower pole calyx. Case 1-EC.

Figure 2b: Hypertrophied papilla of the left upper pole calyx. Case 2-AS.

in normal limits (Supplement 1). Urinalysis demonstrated blood in the urine without proteinuria or signs of infection. Imaging studies included a normal-appearing renal ultrasound as well as a non-contrast $\mathrm{CT}$ revealing fullness in the left renal pelvis. Biopsy of the kidney was unremarkable for renal pathology, such as glomerulonephritis.

The patient underwent cystoscopy with bilateral retrograde pyelogram that revealed efflux of bloody urine from the left ureteral orifice, and filling defects in the left renal pelvic and lower pole calyx (Figure 1). The ureter was stented with a double J stent and subsequent ureteroscopy was performed. The patient was found to have renal papillae which were significantly hypertrophied and highly vascularized (Figure 2). The papilla of the lower pole calyx was hypertrophic to the point of protrusion into the renal pelvis. These hypertrophied lesions were biopsied and then ablated with a holmium laser. Pathology revealed benign fragments of renal medulla with tubular calcifications. 

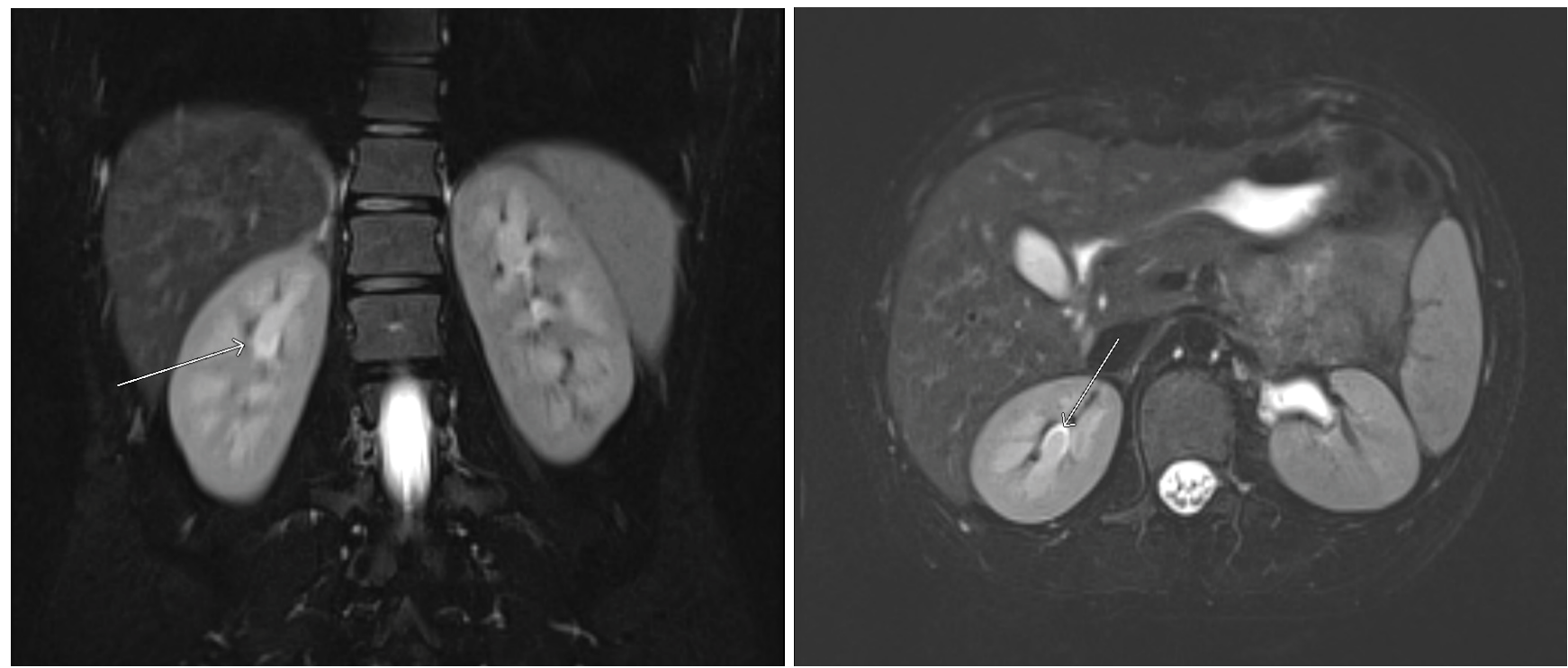

Figure 3: MR Urogram showing prominent bilateral upper pole medullary pyramids consistent with papillary hypertrophy. Case 1-EC.

MR angiogram performed one month later showed no vascular lesions or malformations. MR urogram showed prominent medullary pyramids in bilateral upper poles, consistent with renal papillary hypertrophy (Figure 3). The patient was followed without recurrence of hematuria for over 9 months.

\section{Case 2 - AS}

AS is a 19-year-old female who presented with a 6-month history of persistent, painless gross hematuria that occurred daily. Her initial presentation included dysuria, urinary frequency, and hematuria with unremarkable physical exam. She was treated at that time with a full ten-day course of antibiotics with resolution of all symptoms excluding hematuria. This was initially thought to be due to distention injury of the bladder as the patient voided only 2-3 times per day consistent with dysfunctional voiding; however, the hematuria persisted despite behavioral changes. Additional patient history only elucidated implantation of an etonogestrel implant for contraception five months prior to symptom onset, but no other new medications or exposures. Work-up included complete blood count, coagulation studies, electrolytes, urea nitrogen, creatinine, and hematologic studies for sickle-cell disease which were all within normal limits (Supplement 2). Urinalysis demonstrated RBCs but no protein or infection. Renal ultrasound was normal without masses or cysts.

Patient underwent cystoscopy with retrograde pyelogram that revealed a filling defect in the upper pole calyx of the left kidney, but no lesion in the bladder or contralateral kidney (Figure 1b). A double J stent was placed with delayed ureteroscopy. On ureteroscopy, all the calyces of the left kidney were congested, enlarged, and demonstrated hypertrophic papilla (Figure 2b). Active oozing of blood was seen from the papillae in the upper pole calyx, which was ablated with a holmium laser. Patient was free of hematuria on follow-up for one month. She had recur- rence of hematuria with MRI and MR urogram negative for pathology other than bilateral papillary hypertrophy. Patient's symptom of hematuria resolved and she is asymptomatic for 9 months.

\section{Discussion}

Essential hematuria is a rare condition with poorly understood etiology in many cases. Our patients represent the first cases of bilateral renal papillary hypertrophy as the etiology of essential hematuria.

Papillary hypertrophy is a rare diagnosis since the majority of cases are asymptomatic. Lauret (1956) reported a 9-month-old female who presented with recurrent infections. Retrogrades revealed distortion of the upper pole calyx, and final pathology following nephrectomy revealed a large pyramid with normal histology [4]. Moonen and De Groote (1961) later described two cases of a 25 -year-old female and 11-year-old male who both presented with complains of pain. The female had microscopic hematuria and the boy had a normal urinalysis. These patients underwent partial nephrectomy with pathology revealing hypertrophy of two upper papillae [5]. Whitaker, et al. described the fourth case of hypertrophied papilla. Patient was a 21-year-old male who developed transient episodes of hematuria following dental extraction. Physical examination and laboratory data were unremarkable. Intravenous pyelogram revealed a filling defect in lower pole calyx of right kidney, which was confirmed on retrograde pyelogram. The patient underwent a nephrectomy and pathology revealed a $2 \mathrm{~cm}$-long papilla in the lower pole calyx with normal histology [6].

The etiology of papillary hypertrophy is unknown, and the possibility of hemangiomas of the renal papilla needs to be considered. Both our patients were on hormonal contraception. There may be variation in the hormonal milieu with the use of various birth control treatments which may be associated with papillary hypertrophy or heman- 
gioma of the papilla. Further study on the etiology of this phenomenon is indicated.

Based on the previously reported case reports and our cases, it appears there is no one particular presenting feature of hypertrophied papillae. It is a rare variant that can sometimes cause essential hematuria. In our first case, the MR urogram revealed bilateral hypertrophied medullary pyramids; however, hematuria was unilateral. If the patient were to develop recurrence, a plausible explanation would be further growth and bleeding from the other papillae. Unfortunately, it is difficult to differentiate from upper tract tumors on radiographic studies, and thus requires an invasive and thorough work-up.

\section{Conclusion}

Papillary hypertrophy is a rare abnormality that can result in hematuria. Bilateral involvement has not been reported previously. This is the first report of bilateral hypertrophy by radiologic imaging confirmation. It remains unclear how many asymptomatic patients have radiologically provable papillary hypertrophy. It may be asymptomatic, lead to recurrent infections, or hematuria as shown in our two cases. In our cases, the hypertrophy was noted diffusely by radiologic studies, but required endoscopic evaluation for diagnosis and exclusion of neoplasm of the upper tract. Although the role of hormones is unclear at this time, since both our patients were on hormonal contraception, this entity should be considered in the differential diagnosis of adolescents who present with hematuria. Historically, a nephrectomy or partial nephrectomy was performed in these cases due to lack of correct preoperative diagnosis. However, our case report emphasizes that a minimally invasive approach for diagnosis and management should be the treatment of choice and can result in resolution of hematuria with renal preservation.

\section{References}

1. Livaditis A, Ericsson NO (1962) Essential Hematuria in Children-Prognostic Aspects. Acta Paediatr 51: 630-634.

2. Tanimoto R, Kumon H, Bagley DH (2017) Development of Endoscopic Diagnosis and Treatment for Chronic Unilateral Hematuria: 35 Years Experience. J Endourol 31: S76-S80.

3. Dooley RE, Pietrow PK (2004) Ureteroscopy for benign hematuria. Urol Clin North Am 31: 137-143.

4. Lauret G, Lamy M, Thieffry S, Martin C, Debré R (1956) Urologie de l'enfance. Expansion Scientifique Française, Paris.

5. Moonen WA, DE Groote F (1961) Papillary hypertrophy. S Acta Urol Belg 29: 347-350.

6. Whitaker RH, Edwards D (1969) Congenital hypertrophy of a renal papilla. $\mathrm{Br} \mathrm{J}$ Urol 41: 287-289.

\section{Supplement 1:}

CBC: WBC $11.8 \times 10^{3} /$ microliter, Hgb/Hct $14.1 \mathrm{gm} /$ $\mathrm{dL} / 40.4 \%$, Plt $254 \times 10^{3} /$ microliter

BMP: NA $140 \mathrm{mmol} / \mathrm{L}, \mathrm{K} 3.8 \mathrm{mmol} / \mathrm{L}, \mathrm{Cl} 103 \mathrm{mmol} / \mathrm{L}$, CO2 $22 \mathrm{mmol} / \mathrm{L}$, BUN $12 \mathrm{mg} / \mathrm{dL}, \mathrm{Cr} 0.6 \mathrm{mg} / \mathrm{dL}$, Glucose $86 \mathrm{mg} / \mathrm{dL}$, Ca $10.1 \mathrm{mg} / \mathrm{dL}$

Complement 3104 mg/dL

Complement $419.9 \mathrm{mg} / \mathrm{dL}$

Urine total protein $29 \mathrm{mg} / \mathrm{dL}(0-29 \mathrm{mg} / \mathrm{dL})$

Urine creatinine $208.4 \mathrm{mg} / \mathrm{dL}(12.8-260.8 \mathrm{mg} / \mathrm{dL})$

Protime/INR $10.8 \mathrm{sec} / 1.01$ index

PTT $26.3 \mathrm{sec}$

\section{Supplement 2:}

CBC: WBC $6.9 \times 10^{3} /$ microliter, Hgb/Hct $11.0 \mathrm{gm} /$ $\mathrm{dL} / 34.9 \%$, Plt $390 \times 10^{3} /$ microliter

BMP: NA $142 \mathrm{mmol} / \mathrm{L}, \mathrm{K} 4.2 \mathrm{mmol} / \mathrm{L}, \mathrm{Cl} 105 \mathrm{mmol} / \mathrm{L}$, CO2 $29 \mathrm{mmol} / \mathrm{L}$, BUN $12 \mathrm{mg} / \mathrm{dL}$, Cr $0.8 \mathrm{mg} / \mathrm{dL}$, Glucose $87 \mathrm{mg} / \mathrm{dL}$, Ca $9.8 \mathrm{mg} / \mathrm{dL}$

Urine creatinine $200.6 \mathrm{mg} / \mathrm{dL}(12.8-260.8 \mathrm{mg} / \mathrm{dL})$

Protime/INR $11.2 \mathrm{sec} / 1.06$ index

PTT $24.0 \mathrm{sec}$

Sickle cell screen - Negative 